\title{
Development of the non-linear curvature Wavefront Sensor
}

\author{
Mala Mateen ${ }^{1}$, Johanan Codona ${ }^{2}$, and Olivier Guyon ${ }^{2,3}$ \\ 1 Air Force Research Lab \\ 2 University of Arizona \\ 3 Subaru Telescope, National Observatory of Japan
}

\begin{abstract}
The non-linear Curvature Wavefront Sensor (nlCWFS) is derived from the successful curvature wavefront sensing principle but uses a non-linear reconstruction algorithm to maintain sensitivity to low order modes. It can deliver diffraction-limited images in the visible. Use of the full spatial coherence of the pupil allows the nlCWFS $\left(D / r_{0}\right)^{2}$ gain in sensitivity, over the SHWFS, at the lowest spatial frequency. When background limited the nlCWFS sensitivity scales as $D^{4}$, a combination of $D^{2}$ gain due to the diffraction limit and $D^{2}$ gain due to the telescope's collecting power. For a $30 \mathrm{~m}$ telescope (TMT) with a seeing of $0.5^{\prime \prime}$, the nlCWFS requires 22, 500 fewer photons than the SHWFS to deliver the same wavefront measurement accuracy at the lowest spatial frequencies.
\end{abstract}

\section{Introduction}

The non-linear Curvature Wavefront Sensor (nlCWFS) is derived from the Curvature Wavefront Sensor (CWFS) proposed by François Roddier in 1988 [1] to measure the curvature of the wavefront instead of its slope. The principle of this sensor is presented in Figure 1. A telescope of focal length $f$ images the source in its focal plane, labeled as the nominal focus. A local wavefront curvature that produces an excess of illumination in a plane before the focus will produce a lack of illumination in a plane after the focus. The CWFS consists of two detectors placed in the out of focus planes referred to as Fresnel planes. One detector records the irradiance distribution in the plane of excess illumination and the second records the irradiance distribution in the plane where there is a lack of illumination.

The two Fresnel planes can also be placed on either side of the pupil plane; both representations are equivalent and related to each other through a Fourier transform. Hence the light intensity is measured in planes optically conjugated on either sides of the pupil [2]. CWFSs rely on Fresnel propagation over distance $z$ to transform phase aberrations into intensity fluctuations. The propagation of the complex field on either side of the pupil and the contrast obtained between the two fields is derived in [3][4]. These papers show that contrast can be related to the Laplacian of the wavefront, only in the linear regime when the phase error is less than 1 radian.

\section{2 non-linear Curvature Wavefront Sensor Concept}

The nlCWFS moves away from relating the curvature of the wavefront to the contrast measured by the detector. The nlCWFS is therefore not constrained by the linearity regime in which it is mathematically permissible to relate the Laplacian of the wavefront to the recorded contrast. The nlCWFS extracts information from speckles that develop as phase is propagated from the pupil plane. Figure 2 shows the propagation of a complex field away from the pupil plane along direction $z$. The pupil diameter is $1.5 \mathrm{~m}$ and the field wavelength is $790 \mathrm{~nm}$. The column of images on the right side show intensity measurements made in different Fresnel planes. The Fresnel planes close to the pupil show tight speckles which encode high spatial frequencies and convert phase into intensity at relatively small propagation distances. Fresnel planes further away from the pupil show diffused speckles which encode low spatial frequencies, that are converted into signal at relatively larger propagation distances. In Fig. 2 at $z \approx 22 \mathrm{~km}$ we can see that the light rays begin to cross over one another. In this domain the points in the image plane can no longer be traced back to points in the pupil plane. This is the beginning of 
Give the exact title of the conference

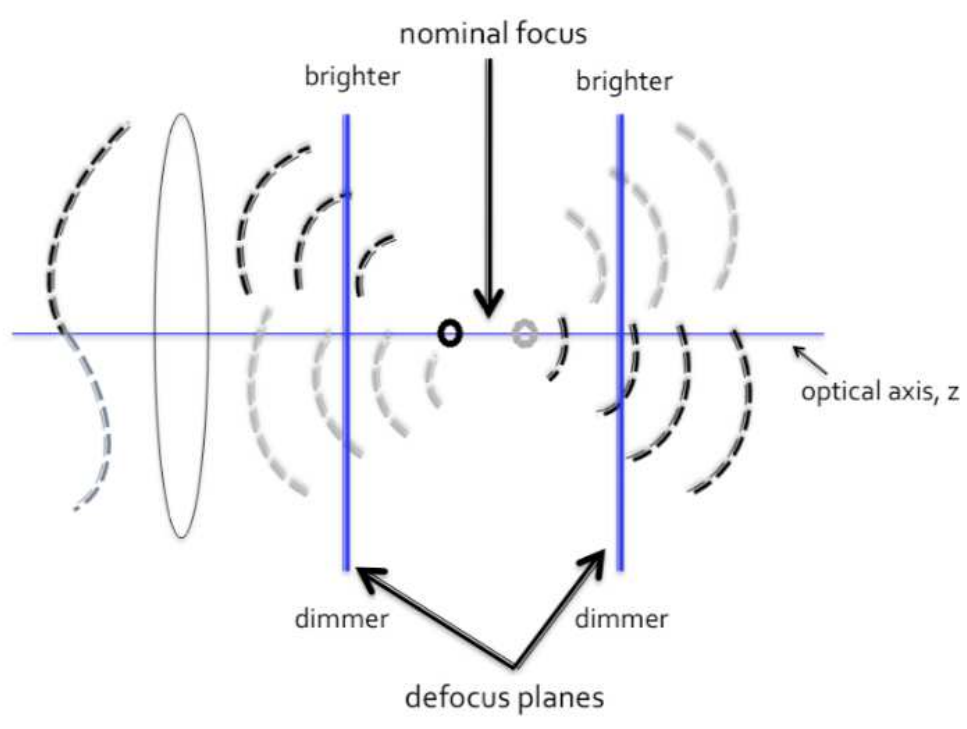

Fig. 1: Principle of curvature wavefront sensing proposed by Roddier [1]. As the wavefront passes through nominal focus its curvature reverses sign. A detector placed on either side of the focus can record the intensity of the wavefront in the two planes.

the non-linear regime where the shape of the wavefront is no longer linearly related to the contrast obtained in conjugate planes on either side of the pupil.

The nICWFS is able to sense low and high spatial frequencies simultaneously by recording the pupil plane wavefront in different Fresnel planes and uses a Gerchberg-Saxton reconstruction algorithm to recover the wavefront in the pupil plane. The Fresnel planes can be selected as far as the lowest spatial frequency, and as close as the highest spatial frequency present in the wavefront. Any number of Fresnel planes can be selected, however we limit them to four to make the reconstructor feasible for a real time adaptive optics system. Four Fresnel planes, that represent the range of spatial frequencies present in the pupil plane wavefront, are selected. The four Fresnel planes can be positioned at any desired distance with respect to the pupil and need not be conjugated on either side of the pupil plane.

The non-linearity of the proposed wavefront sensor imposes a significant penalty on the speed of the the AO control loop. Boot-strapping off of a traditional, linear CWFS could potentially expedite wavefront correction as the initial compensation is done linearly. The linear algorithm would correct all phase aberrations in the $\approx 1$ radian domain before passing on the less aberrated wavefront to the time consuming, non-linear algorithm used by the nlCWFS. The non linear Gerchberg-Saxton reconstruction process is described in section 3. In order to boot-strap off of a traditional, linear CWFS, conjugate pairs of Fresnel planes need to be selected on either side of the pupil plane. The optical design developed later abandons the selection of conjugate pairs due to limited optical-bench space and constraints imposed by chromatic compensation.

\section{Waveoptics Simulations}

Theoretical results based on perturbation analysis, carried out by Guyon [5] suggests that the nlCWFS is significantly more sensitive than the SHWFS at low spatial frequencies. To test the theory and compare the sensitivity of the nlCWFS with the SHWFS we carry out wave-optics simulations in which a $790 \mathrm{~nm}$ electromagnetic field originates from a point source, at a distance of $10^{5} \mathrm{~m}$, and is propagated to the telescope. The size of the telescope aperture is $D=1.5 \mathrm{~m}$. The number of photons simulated for wavefront sensing is $6.7 \times 10^{4}$, which corresponds to $0.1 \mathrm{~ms}$ integration time on a magnitude 10 


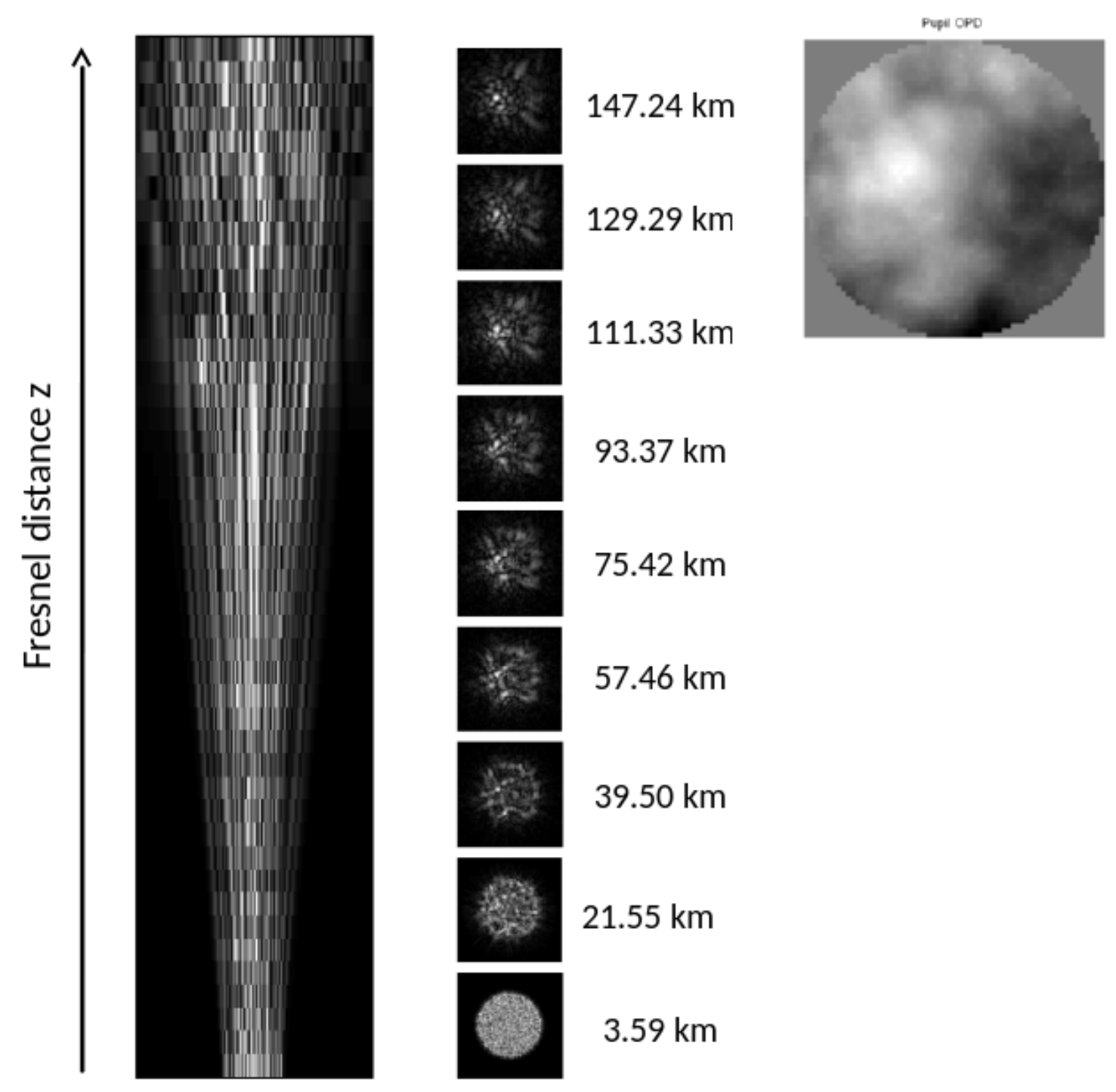

Fig. 2: The diverging light rays show how the rays become non-linear at large Fresnel distances. The arrow points away from the pupil plane and represents Fresnel distance. The column of images on the right show the speckles recorded at different Fresnel distances. High spatial frequencies encoded in tight speckles are converted to signal at a relatively small propagation distance, where the signal is linear. Low spatial frequencies encoded in diffused speckles are converted to signal at relatively larger propagation distances. The signal becomes non-linear at $z \approx 22 \mathrm{~km}$ where the light rays begin to cross over each other. The pupil plane OPD is shown in the top right corner. 
source in a $0.16 \mu \mathrm{m}$ wide bandwidth with $20 \%$ system efficiency. The simulated electromagnetic field consists of $256 \times 256$ grid points spread across $4 \times D ; 64 \times 64$ of the central grid points have phase information. Two phase screens are used to simulate a two-layer atmosphere prescribed with Kolmogorov statistics. Fried's coherence length $r_{0}$ for the two screens is the same but the $C_{n}^{2}$ profile is integrated over different path lengths. The Bufton wind profile is used and the velocity is set to $12.5 \mathrm{~ms}^{-1}$. An example of realistic atmospheric parameters for the Starfire Optical Range is: atmospheric coherence length $r_{0}=0.075 \mathrm{~m}$ and isoplanatic angle $\theta_{0}=9.4 \mu \mathrm{rad}$ at $500 \mathrm{~nm}$. According to the scaling formulas given in Eq. 1 and Eq. 2 these atmospheric parameters translate to $D / r_{0}=12.7$, and $\theta_{0}=16.2 \mu \mathrm{rad}$ at $790 \mathrm{~nm}$ when looking at zenith [6].

$$
\begin{aligned}
& r_{0}\left(\lambda_{2}\right)=r_{0}\left(\lambda_{1}\right)\left(\frac{\lambda_{2}}{\lambda_{1}}\right)^{6 / 5} \\
& \theta_{0}\left(\lambda_{2}\right)=\theta_{0}\left(\lambda_{1}\right)\left(\frac{\lambda_{2}}{\lambda_{1}}\right)^{6 / 5}
\end{aligned}
$$

The Greenwood frequency at $790 \mathrm{~nm}$ is $f_{g}=171 \mathrm{~s}^{-1}$. The tilt removed wavefront distortion for Kolmogorov turbulence averaged over an aperture of diameter $D$ expressed as a root-mean-square (RMS) value in units of radians of optical phase is [6]:

$$
<\phi>=0.376\left(\frac{D}{r_{0}}\right)^{5 / 6}
$$

According to Eq. 3 for $D=1.5 \mathrm{~m}$ and $r_{0}=0.12 \mathrm{~m}$ a realistic wavefront will have 3 radians of RMS error. The wavefront simulated for the sensitivity analysis has an RMS error equal to 3.48 radians and is shown in Fig. 3. The simulation parameters are listed in Table 1. Simulations assume both detectors to be perfect with $100 \%$ quantum efficiency and zero read noise of. The only source of error in the simulations is photon noise.

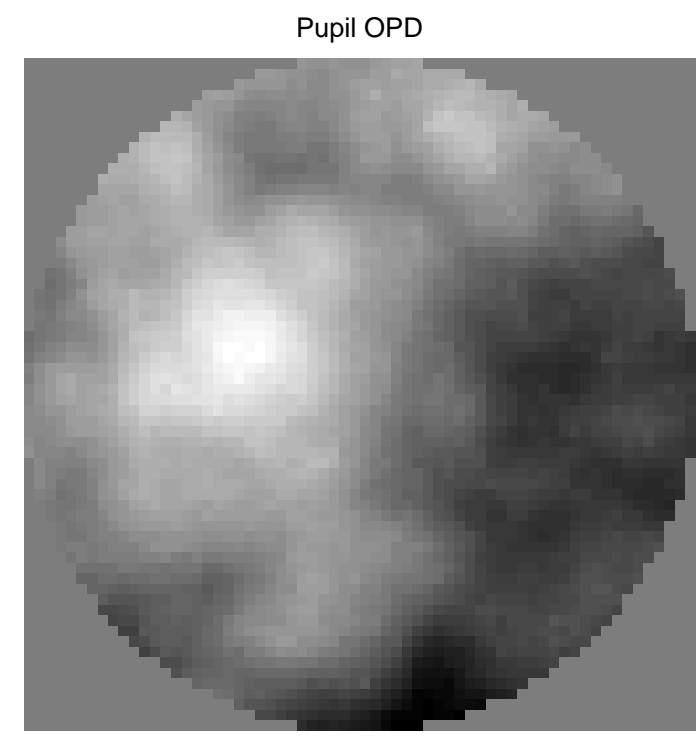

Fig. 3: The simulated pupil plane wavefront after tilt removal has an RMS error of 3.48 radians. 
Table 1: Simulations Parameters

\begin{tabular}{|c|c|c|}
\hline Parameter & nlCWFS & SHWFS \\
\hline Telescope diameter & $1.5 \mathrm{~m}$ & \\
\hline WFS wavelength & $790 \mathrm{~nm}$ & \\
\hline RMS wavefront error & $3.48 \mathrm{rad}$ & \\
\hline Integration time & $0.001 \mathrm{~s}$ & \\
\hline Source brightness & $6.7 \times 10^{4}$ photons & \\
\hline Fried's coherence length & $0.12 \mathrm{~m} @ 790 \mathrm{~nm}$ & \\
\hline Isoplanatic angle & $16.2 \mu \mathrm{rad} @ 790 \mathrm{~nm}$ & \\
\hline Greenwood frequency & $171 \mathrm{~s}^{-1} @ 790 \mathrm{~nm}$ & \\
\hline WFS detector readout noise & 0 & \\
\hline WFS detector quantum efficiency & 1 & \\
\hline WFS subapertures & not applicable & 32 \\
\hline Fresnel plane distances & $287 \mathrm{~km}, 575 \mathrm{~km}, 862 \mathrm{~km}, 1149 \mathrm{~km}$ & not applicable \\
\hline WFS detector sampling & 64 pixels across pupil & $2 \times 2$ pixels per subaperture \\
\hline Spatial frequency control range & $\begin{array}{l}16 \mathrm{CPA} \text { at } 2 \times \text { Nyquist } \\
32 \mathrm{CPA} \text { at Nyquist }\end{array}$ & $\begin{array}{ll}8 \mathrm{CPA} \text { at } & 2 \times \text { Nyquist } \\
16 \mathrm{CPA} \text { at Nyquist } & \end{array}$ \\
\hline
\end{tabular}

\section{Gerchberg-Saxton Reconstruction}

For simplicity We have chosen to implement a Gerchberg-Saxton [7] non-linear reconstruction algorithm, however other reconstruction algorithms exist. A Gerchberg-Saxton iterative loop is used to reconstruct the phase sensed with the nlCWFS. The Gerchberg-Saxton reconstruction algorithm, illustrated in Figure 4 works by propagating complex fields between planes to recover the phase information in the pupil plane. The propagation is carried out by computing the Fresnel diffraction integral which is derived in [3].

The GS algorithm requires high resolution which translates to large number of camera pixels, which in turn equates to large shot noise. As the source gets dimmer $\left(m_{v} \geq 9\right)$ the GS reconstruction degrades because now fewer photons are distributed over a set number of pixels lowering the SNR. It the number of pixels is reduced then there is not enough resolution for the GS algorithm to extract all the spatial frequencies and the unsensded modes show up as focus. Therefore an alternative reconstruction algorithm is required. In section 6 we discuss the development of a non-iterative, real-time, non-linear reconstruction algorithm. Reconstruction of a simulated wavefront using the GS algorithm is show in Figure 5.

\section{Sensitivity Comparison}

The following formula relates two physical quantities, $\Sigma$ and $N$ through the scalar $\beta$ [8],

$$
\beta=\Sigma * \sqrt{N}
$$

here $\Sigma$ is the error per Fourier mode given in radians, $N$ is the number of photons incident on the detector, and $\beta$ represents the sensitivity of the wavefront sensor to a Fourier mode. If photon noise is the only contributing source of error then the error per Fourier mode given a signal-to-noise-ratio (SNR), can be determined. For a fixed SNR a lower error or $\Sigma$ implies a lower $\beta$ which in turn implies that the WFS requires fewer photons to reconstruct a Fourier mode and is thus a more sensitive WFS.

A Monte Carlo experiment is conducted in which the pupil plane wavefront is generated a thousand times, with photon noise being the only variable parameter between iterations. The thousand wavefronts are reconstructed with each of the sensors and then decomposed into Fourier modes. The standard deviation of the Fourier mode amplitude is determined over the thousand iterations and gives the error per mode, which is $\Sigma$ from Eq. 4. Details of the Fourier decomposition, Fourier mode coupling, and error per Fourier mode are presented in a separate paper [9], currently underway. In the case 
Pupil OPD
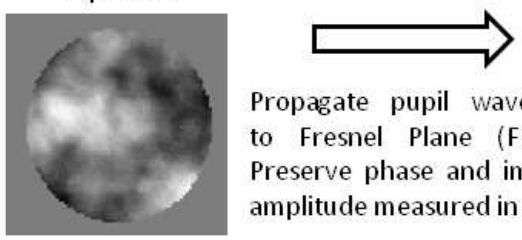

Propagate pupil wavefront to Fresnel Plane (FP) 1. Preserve phase and impose amplitude measured in FP1.

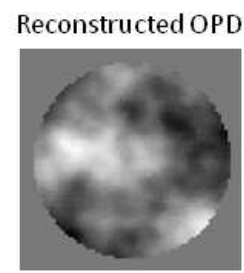

Fresnel Plane 1
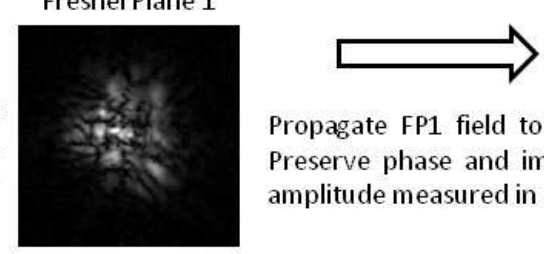

Propagate FP1 field to FP2. Preserve phase and impose amplitude measured in FP2.
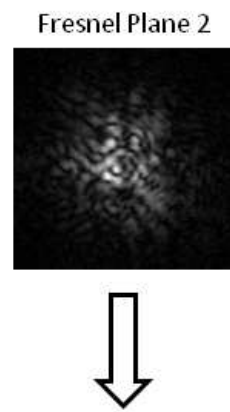

Fresnel Plane 3

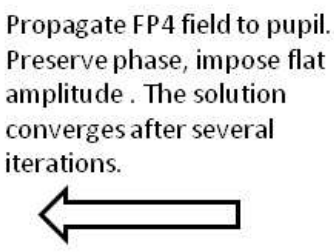

Fresnel Plane 4

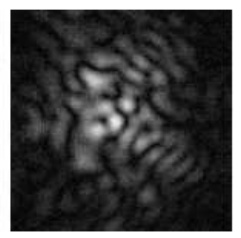

Propagate FP3 field to FP4.

Preserve phase and impose amplitude measured in FP3.

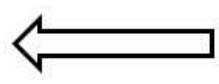

Fig. 4: The Gerchberg-Saxton reconstruction algorithm is illustrated. The pupil plane optical path difference (OPD) shown in the top right corner is propagated to Fresnel plane (FP) 1 where the phase acquired due to propagation is preserved and the amplitude thrown out. The amplitude measured at FP 1 is imposed and a complex field is constructed using the propagated phase and the measured amplitude. The complex field in FP 1 is propagated to FP 2. A complex field similar to the one constructed in FP1, is constructed at each of the consecutive FPs and the field at the final FP (here FP 4) is propagated back to the pupil plane. At the pupil plane a flat field is imposed. It takes several iterations of the Gerchberg-Saxton loop to converge to the pupil plane phase.

of the nlCWFS, photon noise is added at each Fresnel plane. For the SHWFS, photon noise is added at a single detector plane. The detectors are modeled to be noise free and have a quantum efficiency of 1 , therefore the only source of error is photon noise. For the sensitivity analysis we want to determine the error or standard deviation, due to photon noise, in reconstructing each Fourier mode. We compute the standard deviation of the amplitude of the 3113 Fourier modes over a thousand iterations and compute $\beta$ per spatial frequency for the nICWFS and the SHWFS. The number of photons needed to reconstruct each Fourier mode to 1 radian RMS is equal to $\beta^{2}$. For the nlCWFS and the SHWFS $\beta^{2}$ is plotted as a function of spatial frequency in Figure 6. The Wavefront sensor sensitivity is determined for up to 16 spatial frequencies. The simulated results show that at the lowest spatial frequency the nlCWFS requires $\approx 71$ times fewer photons than the SHWFS. On average the nlCWFS requires 12 times fewer photons than the SHWFS to reconstruct spatial frequencies of 1 to 10 cycles per aperture (CPA). Theoretical estimates based on perturbation analysis predict that, on average, the nlCWFS requires $\approx 14$ times fewer photons than the SHWFS to reconstruct the first 10 spatial frequencies [5]. The simulated results agree well with the theoretical predictions. Note that the source brightness and other parameters used in the theoretical analysis slightly differ from the more realistic, site, and telescope specific parameters used in the simulations. For instance the theoretical source is much brighter than the more realistic source used in the simulations, making the simulated source brighter will only improve the results as the error per mode will decrease. The theoretical RMS wavefront error is $\approx 4$ radian compared to the 3.48 radian used for the simulations; the small difference does not effect the results. The theoretical wavelength is $850 \mathrm{~nm}$ compared to the $790 \mathrm{~nm}$ used in simulations, and the telescope diameter used in the theoretical analysis is $8 \mathrm{~m}$ compared to the $1.5 \mathrm{~m}$ used in simulations. Despite the minor differences the sensitivity analysis can be compared for similar RMS wavefront errors. 

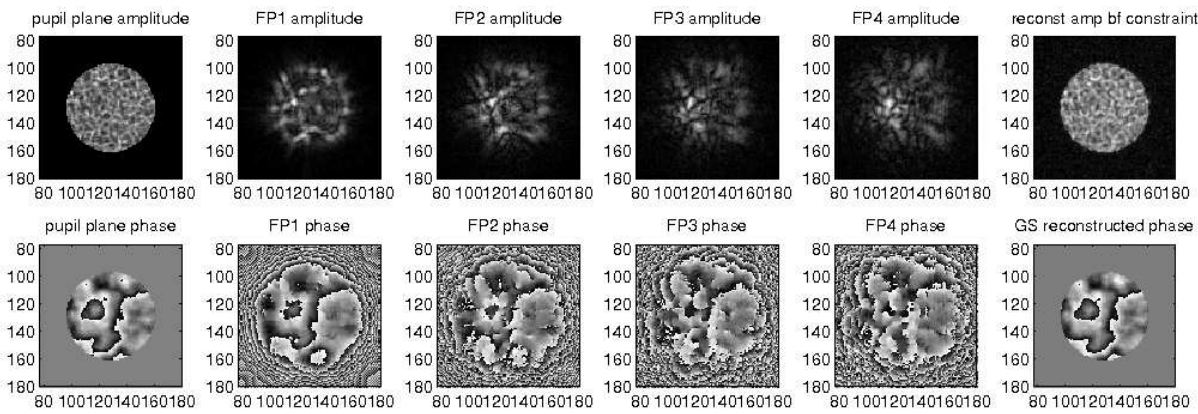

FP1 phase

FP2 phase
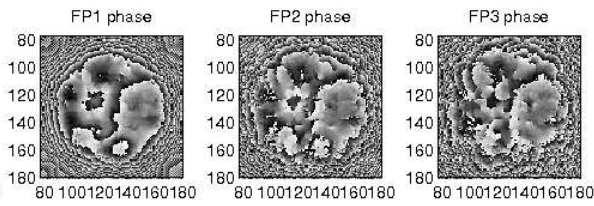

FP4 phase
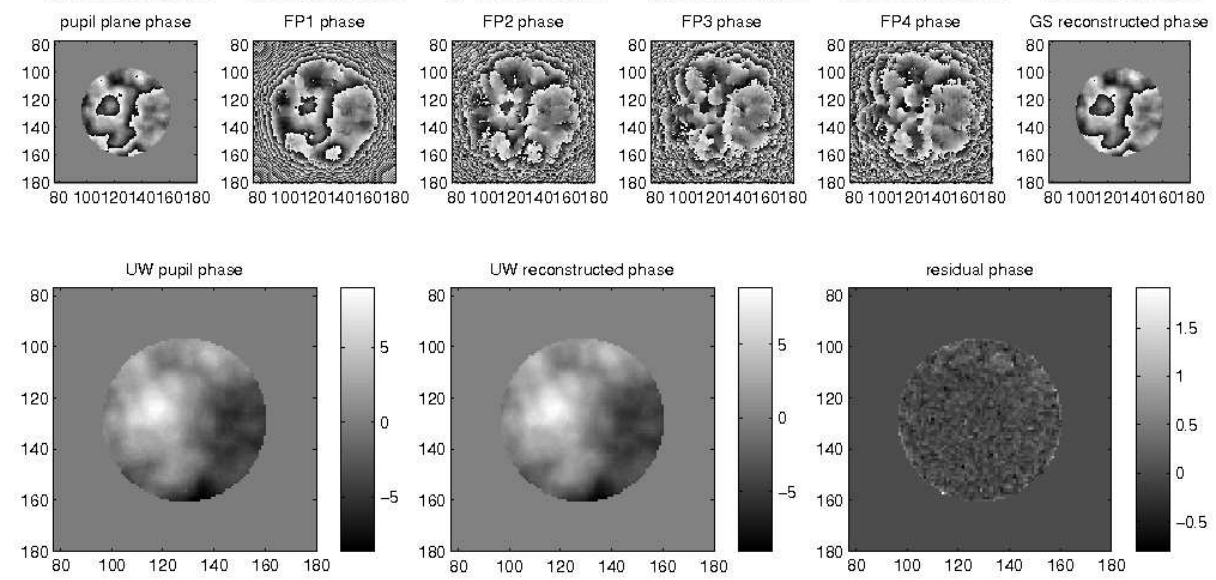

Fig. 5: nlCWFS Gerchberg-Saxton reconstruction. The top row shows the amplitude in the pupil and at each of the Fresnel planes. The middle row shows the phase in the pupil and in each of the Fresnel planes. The third row shows the input phase, the reconstructed phase and the residual between the two. Reconstruction after 50 iterations of the Gerchberg-Saxton loop is shown.

The results obtained are quite encouraging and indicate that using the nlCWFS instead of the SHWFS will make it possible to observe dimmer exoplanets, as the nlCWFS requires significantly fewer photons to sense the wavefront.

\section{Future Work}

Simulations comparing the strrehl obtained by closing the loop on the nlCWFS and the SHWFS, show an improved performance for the nlCWFS for objects that are $m_{v}<9$. For dimmer $\left(m_{v} \geq 9\right)$ objects the GS reconstruction algorithm is unable to reconstruct the wavefront. The GS algorithm requires a high spatial resolution which means the Fresnel images contain a large number of pixels. Having a large number of pixels leads to higher shot noise. The shot noise increases as the object gets dimmer and fewer of the diffracted rays reach the Fresnel planes. If the number of pixels in the Fresnel planes is reduced, it helps lower the shot noise but also lowers the resolution making it difficult for the GS algorithm to extract all the spatial frequencies in the pupil. These unsensed spatial frequencies show up as focus.

Due to the limitations of the GS algorithm we are exploring an alternative real-time reconstructor. The alternative reconstructor represented in Figure 7 is based on concepts from weak-through-strong scintillation theory. Even if the scintillations incident on the telescope are negligible, phase aberrations in the pupil plane cause scintillation inside the optical system, increasing from weak to strong as the light propagates towards the focal plane. The important scales are the Fried length $r_{0}$, the Fresnel scale $R_{f}=(\lambda z)^{1 / 2}$ where $\mathrm{z}$ is the propagation distance from the pupil, and the pupil diameter $\mathrm{D}$ which limits the maximum spatial scale. Near the pupil plane, $R_{f} \ll r_{0}$, irradiance fluctuations are 
Give the exact title of the conference

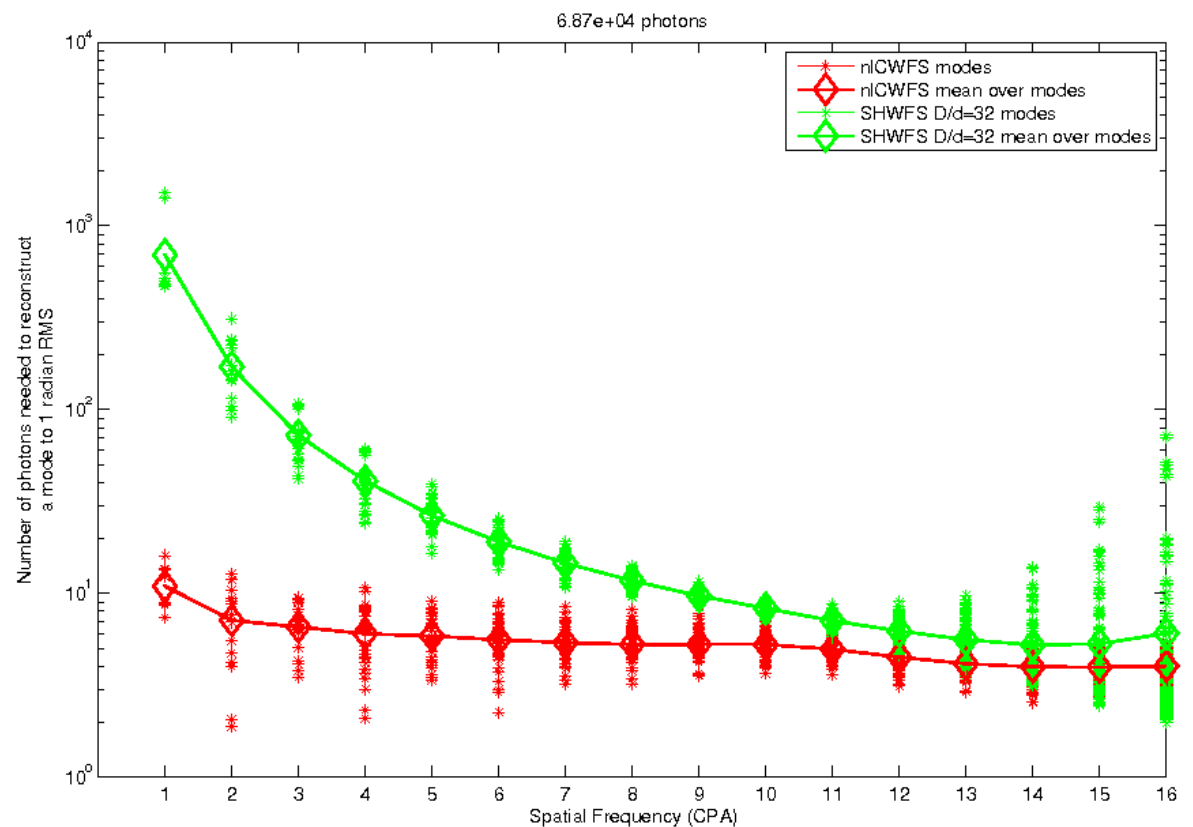

Fig. 6: Comparison of the nlCWFS sensitivity with the SHWFS. The number of photons needed to reconstruct a mode to 1 radian RMS is plotted as a function of the CPA, for up to 16 spatial frequencies. The sensitivity curve for the nlCWFS is shown in red and that for the SHWFS is shown in green. The * indicate the number of photons needed to reconstruct the modes. The $*$ represent the individual modes present in a spatial frequency. The diamond show the mean of the modes over the particular spatial frequencies.

weak and proportional to pupil plane wavefront curvature. At this range irradiance deviations from the mean provide a means for measuring the small-scale aberrations, but are insensitive to larger-scale aberrations. As we move farther from the pupil plane, scintillations increase in strength and exhibit both small and larger-scale structure. In general, the large-scale scintillation modulates the small-scale features. The mean irradiance PSD of the large-scale scintillation is described by the low-frequency series in [10]. Although the smaller-scale features are more complicated than this, if we subtract the mean irradiance profile and smooth over the smaller features, we are left with just the modulating profile, which is refractive in nature and is also sensitive to wavefront curvature, but on a larger scale. Eventually, as we move away from the pupil, the Fresnel scale becomes comparable or larger than D and we are limited from seeing larger scales by the pupil. Here the largest aberrations appear as tip-tilt motion in the beam, while defocus and astigmatism appear as variations in the overall beam width and shape. The tip-tilt can be measured by a centroid (first spatial moment) of the beam, with greater sensitivity achieved by using the more distant planes. The defocus and astigmatism can be measured using the four second-order spatial moments found by integrating over the irradiance. Again, this is more sensitive in the more distant planes, and unambiguous so long as we are never in the focal plane.

The resulting algorithm is summarized as follows. Collect irradiance images at a number of distances behind the pupil. After preprocessing the images, the centroid and second moments are computed to give estimates of tip-tilt, defocus and astigmatism. Each of the irradiance images have their running means subtracted and are smoothed over a size proportional to the Fresnel scale to give a set of "difference images". Each resulting image is proportional to the wavefront curvature, but sensitive to different spatial scales. Since the sensitivities overlap, the mixture will be calibrated and combined into a single matrix that includes the smoothing and the mixing into a single "reconstructor matrix" 
to be multiplied by the pixels of the difference images. Using the result from just this much of the algorithm will give a useful estimate of the pupil plane wavefront, but not yet at the full sensitivity of the nlCWFS. The final step is to use the estimated wavefront to compute the resulting irradiance at the various camera planes and compare them to the measured irradiance. Since the features have spatial scales of $\frac{\lambda z}{D}$, any residuals can be measured to the full sensitivity of the system. One way to utilize this information is to make a single, non-iterative update using the phase from the computed field in one or more Fresnel plane with the square root of the actual image data as the amplitude and propagate back to the pupil plane. Combining the resulting wavefront error estimate with the smoothed difference image contributions, defocus, astigmatism, and tip-tilt, we arrive at the new updates for the DM. These are applied and the process repeats. Since most of these steps can be performed in parallel, the algorithm is highly appropriate for parallel or GPU architectures. 
Give the exact title of the conference
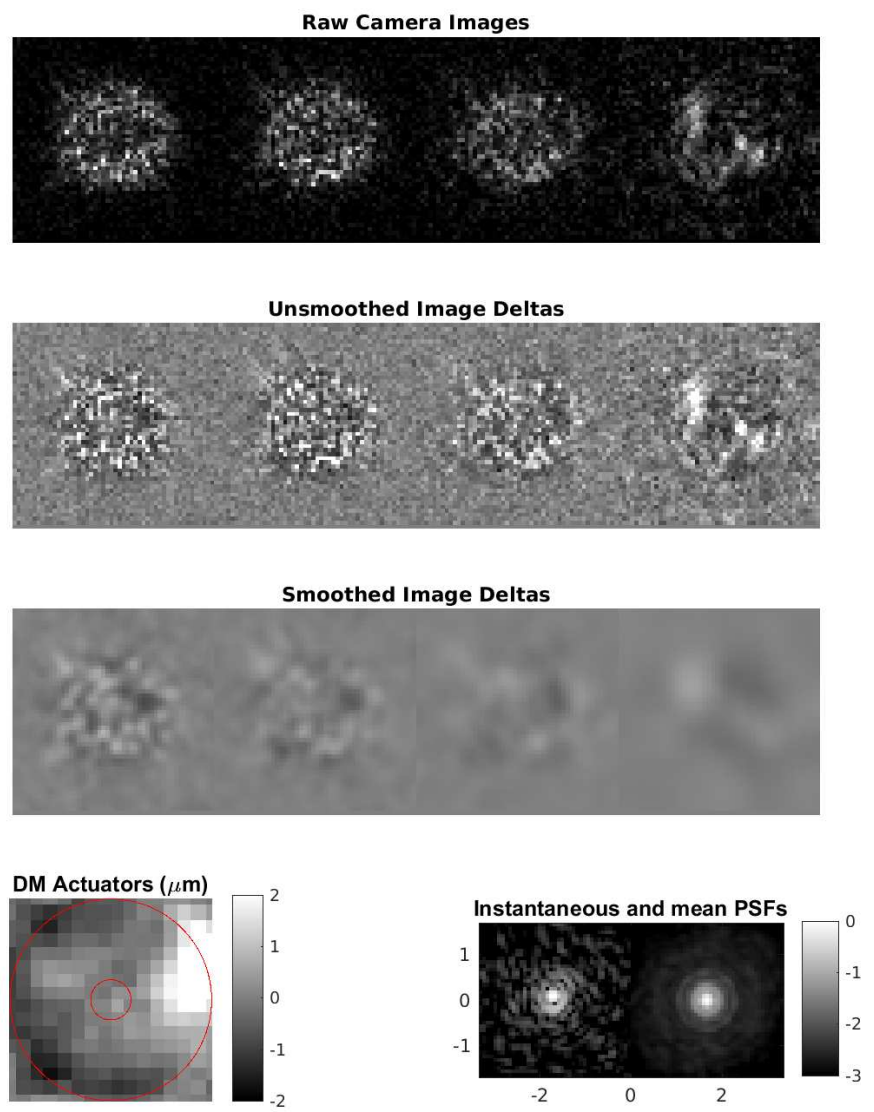

Fig. 7: An example of image plane processing. The top row of images are collected at four different distances from the pupil. A running mean is kept for each plane and is subtracted from the latest images. This results in an estimate of increased or decreased irradiance, shown in the second row. In the third row of images, each image is smoothed by a filter that is more broad for more distant planes. These images are combined with the first and second moments of the irradiance to give the current wavefront error. An optional final step compares the irradiance computed from the estimate with the actually measured irradiance, giving a final correction to the wavefront error to be applied to the DM.

\section{References}

1. F. Roddier. Curvature sensing and compensation: a new concept in adaptive optics. $A O, 27: 1223-$ 1225, April 1988.

2. F. Roddier, M. Northcott, and J. E. Graves. A simple low-order adaptive optics system for nearinfrared applications. PASP, 103:131-149, January 1991.

3. M. Mateen, V. Garrel, M. Hart, and O. Guyon. Results from the laboratory demonstration of the nonlinear curvature wavefront sensor. In Society of Photo-Optical Instrumentation Engineers (SPIE) Conference Series, volume 7736 of Society of Photo-Optical Instrumentation Engineers (SPIE) Conference Series, page 19, July 2010. 
4. O. Guyon. Ultra-high-sensitivity wavefront sensing for extreme-AO. In Society of Photo-Optical Instrumentation Engineers (SPIE) Conference Series, volume 7015 of Presented at the Society of Photo-Optical Instrumentation Engineers (SPIE) Conference, July 2008.

5. O. Guyon. High Sensitivity Wavefront Sensing with a Nonlinear Curvature Wavefront Sensor. PASP, 122:49-62, January 2010.

6. J. W. Hardy. Adaptive Optics for Astronomical Telescopes. Oxford University Press, July 1998.

7. R. W. Gerchberg and W. O. Saxton. A practical algorithm for determination of the phase from image and diffraction planes. Optik, 35:237-246, December 1972.

8. O. Guyon. Limits of Adaptive Optics for High-Contrast Imaging. ApJ, 629:592-614, August 2005.

9. M. Mateen and O. Guyon. Verification of the Non-linear Curvature Wavefront Sensor. Journal of the Optical Society of America (1917-1983), In progress.

10. J.L. Codona, S.M. Creamer, R.G. Frehlich, and Henyey F.S. Solution of the fourth moment of waves propagating in random media. Radio Sci., 21:929-948, 1986. 